\title{
Monotone difference schemes for weakly coupled elliptic and parabolic systems
}

\author{
Piotr Matus* Francisco Gaspar ${ }^{\dagger} \quad$ Le Minh Hieu ${ }^{\ddagger}$ \\ Vo Thi Kim Tuyen ${ }^{\S}$
}

\begin{abstract}
The present paper is devoted to the development of the theory of monotone difference schemes, approximating the so-called weakly coupled system of linear elliptic and quasilinear parabolic equations. Similarly to the scalar case, the canonical form of the vector-difference schemes is introduced and the definition of its monotonicity is given. This definition is closely associated with the property of non-negativity of the solution. Under the fulfillment of the positivity condition of the coefficients, two-side estimates of the approximate solution of these vector-difference equations are established and the important a priori estimate in the uniform norm $C$ is given.
\end{abstract}

Keywords: monotone schemes, maximum principle, weakly coupled elliptic system, two-side estimates, uniform norm, non-uniform grids.

\section{Introduction}

Computational methods satisfying the discrete maximum principle are called monotone [14]. The maximum principle allows us not only to establish unique solvability of the corresponding difference problems, but also to obtain the important theoretical a priori estimates of stability and convergence of the difference solution in the strongest uniform norm $C$ or $L_{\infty}$. Monotone difference schemes play an important role in mathematical modeling applications, because they allow to obtain numerical solutions without nonphysical oscillations [10].

Extensive literature is devoted to the study of monotone difference schemes for linear elliptic and parabolic equations in the scalar case (see $[6,18]$ ), for example. However, due to the difficulty of the problem, similar results for systems of elliptic equations are missing in the scientific literature. This is the case, for example, of the typical elasticity system of PDEs. In this context, it is interesting to note papers $[1,2]$, where monotone finite element methods for parabolic PDE systems are studied.

*Institute of Mathematics and Computer Science, The John Paul II Catholic University of Lublin, Al. Raclawickie 14, 20-950 Lublin, Poland, and Institute of Mathematics, NAS of Belarus, 11 Surganov Str., 20072 Minsk, Belarus, Email: matus@im.bas-net.by

${ }^{\dagger}$ CWI, Centrum Wiskunde and Informatica, Amsterdam, The Netherlands, Email: gaspar@cwi.nl

$\ddagger$ Belarusian State University, 4 Nezavisimosti avenue, 220030 Minsk, Belarus, and University of Economics, The University of Danang, 71 Ngu Hanh Son Str., 590000 Danang, Vietnam, Email: lmhieuktdn@gmail.com

§Belarusian State University, 4 Nezavisimosti avenue, 220030 Minsk, Belarus, Email: vokimtuyen188@gmail.com 
The present paper is dedicated to the development of the theory of monotone difference schemes approximating the so-called weakly coupled system of linear elliptic and quasilinear parabolic equations [11]. Similarly to the scalar case [14] the canonical form of the vector-difference schemes is introduced and the definition of its monotonicity is given. This definition is closely associated with the property of non-negativity of the approximate solution. Under the fulfillment of positivity condition of the matrix coefficients, two-side estimates of difference solution of these vector-difference equations are established and the important a priori estimates in the uniform norm $C$ are given. Note that the twoside estimates are especially important for the analysis of theoretical properties of the computational methods approximating problems with unbounded nonlinearities, where it is necessary to prove that the discrete solution belongs to a neighborhood of the exact solution [8]. An example is the Gamma equation modeling options pricing in financial mathematics [3]. Obtained results are applied to analyze the properties of monotonicity of the difference schemes in second order system of linear elliptic and quasi-linear parabolic equations on non-uniform grids [15].

The outline of the paper is as follows. In Section 2 the canonical form of the vector difference schemes is introduced and the definition of its monotonicity is given. Here, we understand the monotonicity as a corollary of the maximum principle: from nonnegativity (non-positivity) of the input data it follows non-negativity (non-positivity) of all components of the unknown grid vector-function. This definition is closely connected with the works dedicated to finite difference schemes (FDS) preserving positivity of its solution [3]. In this section, the sufficient conditions of positivity of the coefficients are formulated. By using these conditions, two-side estimates of the difference solution of the vector-difference equation and the estimate in the norm $C$ are proved. In Section 3 we consider a FDS on uniform grids for a two-dimensional weakly coupled steady problem without convection. Monotonicity of these difference schemes are studied and a priori estimates of stability of the difference solution are established. In Sections 4 and 5 the obtained results are generalized to two-dimensional weakly coupled linear elliptic systems of convection-diffusion equations and two-dimensional quasilinear systems of parabolic type respectively. Note that in the quasilinear parabolic equation nonlinear coefficient depends on all components and we have built the difference schemes of second order of approximation. To construct unconditionally monotone schemes, the idea of regularization in the scalar case of A. Samarskii [14] is used. Section 6 is dedicated to construct unconditionally monotone vector-difference schemes of second order approximation on an arbitrary non-uniform grid for one-dimensional weakly coupled elliptic systems. For this purpose, the well-known idea of designing such schemes in the scalar case is used $[15,7,9,16,4]$.

\section{Monotonicity of finite difference schemes for sys- tems of PDEs}

In this section we define the concept of monotonicity of finite difference schemes for systems of partial differential equations. Let $\bar{\omega}_{h}=\omega_{h} \cup \partial \omega_{h}$ denote a grid in a bounded domain $\bar{\Omega} \subset R^{n}$, where $\omega_{h}$ and $\partial \omega_{h}$ are the sets of interior and boundary grid points respectively.

To each point $P \in \omega_{h}$ we associate one and only one stencil $M(P)$ - a subset of $\bar{\omega}_{h}$, containing this point. The set $M^{\prime}(P)=M(P) \backslash P$ is called neighborhood of the point $P$. Next, for each inner grid point $P \in \omega_{h}$ we consider the following finite difference scheme 
written in form

$$
A(P) \mathbf{Y}(P)=\sum_{Q \in M^{\prime}(P)} B(P, Q) \mathbf{Y}(Q)+\mathbf{F}(P), \quad P \in \omega_{h}
$$

which is called a canonical form of the vector-difference scheme in a way analogous to the scalar case [14]. Here $\mathbf{Y}(P)=\left(y_{1}(P), y_{2}(P), \ldots, y_{m}(P)\right)^{T}$ is the unknown vector grid function associated with node $P, A(P)=\left(a_{i j}(P)\right)_{m \times m}$ and $B(P, Q)=\left(b_{i j}(P, Q)\right)_{m \times m}$ are the matrices of coefficients of the equations, $\mathbf{F}(P)=\left(f_{1}(P), f_{2}(P), \ldots, f_{m}(P)\right)^{T}$ is the right-hand side vector of the system.

In this work we assume Dirichlet boundary conditions for all variables, and therefore, for each boundary point $P \in \partial \omega_{h}$, we have

$$
\mathbf{Y}(P)=\boldsymbol{\mu}(P), \quad P \in \partial \omega_{h},
$$

where $\boldsymbol{\mu}(P)=\left(\mu_{1}(P), \mu_{2}(P), \ldots, \mu_{m}(P)\right)^{T}$ is a given vector. In the sequel the following notation will be used:

$$
\begin{gathered}
\max _{P \in \bar{\omega}_{h}} \mathbf{Y}(P)=\max _{1 \leq k \leq m} \max _{P \in \bar{\omega}_{h}} y_{k}(P), \quad \min _{P \in \bar{\omega}_{h}} \mathbf{Y}(P)=\min _{1 \leq k \leq m} \min _{P \in \bar{\omega}_{h}} y_{k}(P), \\
\|\mathbf{Y}\|_{\bar{\omega}_{h}}=\max _{1 \leq k \leq m} \max _{P \in \bar{\omega}_{h}}\left|y_{k}(P)\right|, \quad\|\mathbf{Y}\|_{\partial \omega_{h}}=\max _{1 \leq k \leq m} \max _{P \in \partial \omega_{h}}\left|y_{k}(P)\right|, \\
A(P) \geq 0 \Leftrightarrow a_{i j}(P) \geq 0 \quad \forall i, j, \quad \mathbf{F}(P) \geq 0 \Leftrightarrow f_{i}(P) \geq 0 \quad \forall i .
\end{gathered}
$$

Definition 1. Finite difference scheme (1)-(2) is called monotone if its solution satisfies the conditions:

$$
\begin{aligned}
& \text { If } \mathbf{F}(P) \geq 0 \forall P \in \omega_{h} \text { and } \boldsymbol{\mu}(P) \geq 0 \forall P \in \partial \omega_{h} \text { then } \mathbf{Y}(P) \geq 0 \forall P \in \bar{\omega}_{h} . \\
& \text { If } \mathbf{F}(P) \leq 0 \forall P \in \omega_{h} \text { and } \boldsymbol{\mu}(P) \leq 0 \forall P \in \partial \omega_{h} \text { then } \mathbf{Y}(P) \leq 0 \forall P \in \bar{\omega}_{h} .
\end{aligned}
$$

We now give sufficient conditions on the coefficients of the equations, that can be checked easily a priori, in order to ensure the monotonicity of the scheme. For this, we rewrite finite difference scheme (1)-(2) as

$$
\begin{aligned}
A_{1}(P) \mathbf{Y}(P) & =\sum_{Q \in M^{\prime}(P)} B(P, Q) \mathbf{Y}(Q)+C_{1}(P) \mathbf{Y}(P)+\mathbf{F}(P), P \in \omega_{h}, \\
\mathbf{Y}(P) & =\boldsymbol{\mu}(P), \quad P \in \partial \omega_{h},
\end{aligned}
$$

where $A(P)=A_{1}(P)-C_{1}(P)$, with $A_{1}(P)$ the diagonal matrix of the diagonal entries of $A, A_{1}(P)=\operatorname{diag}\left(a_{11}(P), a_{22}(P), \ldots, a_{m m}(P)\right)$, and $C_{1}(P)=\left(c_{i j}^{1}(P)\right)_{m \times m}$. If $D(P)=$ $\operatorname{diag}\left(d_{11}(P), d_{22}(P), \ldots, d_{m m}(P)\right)$ is the diagonal matrix of order $m$ defined by

$$
d_{i i}(P)=a_{i i}(P)-\left(\sum_{Q \in M^{\prime}(P)} \sum_{j=1}^{m} b_{i j}(P, Q)+\sum_{j=1}^{m} c_{i j}^{1}(P)\right),
$$

we have the following result in order to guarantee the property of monotonicity of the finite difference scheme.

Theorem 1. If the coefficients of problem (5)-(6) satisfy the positivity conditions

$$
A_{1}(P)>0, \quad B(P, Q) \geq 0, \quad C_{1}(P) \geq 0, \quad D(P)>0,
$$

then the following two-side estimate holds

$$
m_{1} \leq y_{k}(P) \leq m_{2}, \quad k=1, \ldots, m,
$$


where

$$
\begin{aligned}
& m_{1}=\min \left\{\min _{P \in \partial w_{h}} \boldsymbol{\mu}(P), \min _{P \in \omega_{h}}\left(D^{-1}(P) \mathbf{F}(P)\right)\right\}, \\
& m_{2}=\max \left\{\max _{P \in \partial w_{h}} \boldsymbol{\mu}(P), \max _{P \in \omega_{h}}\left(D^{-1}(P) \mathbf{F}(P)\right)\right\},
\end{aligned}
$$

and therefore the finite difference scheme is monotone.

Proof. We prove the upper bound of estimate (8). In a similar way, the lower bound can be proved. If the vector grid function $\mathbf{Y}(P)$ attains its maximum in sense of notation (3) at a boundary point $P_{0} \in \partial \omega_{h}$ then it exists $j \in\{1,2, \ldots, m\}$ such that

$$
\max _{P \in \bar{\omega}_{h}} \mathbf{Y}(P)=\mu_{j}\left(P_{0}\right) .
$$

Let us now suppose that the solution achieves its maximum at an interior grid point $P_{0} \in \omega_{h}$, i.e., it exists $i \in\{1,2, \ldots, m\}$ such that

$$
\max _{P \in \bar{\omega}_{h}} \mathbf{Y}(P)=y_{i}\left(P_{0}\right)
$$

From (5), we have the equation

$$
a_{i i}\left(P_{0}\right) y_{i}\left(P_{0}\right)=\sum_{Q \in M^{\prime}\left(P_{0}\right)} \sum_{j=1}^{m} b_{i j}\left(P_{0}, Q\right) y_{j}(Q)+\sum_{j=1, j \neq i}^{m} c_{i j}^{1}\left(P_{0}\right) y_{j}\left(P_{0}\right)+f_{i}\left(P_{0}\right) .
$$

By applying the positive conditions (7) of the coefficients of the problem, we obtain the following inequality

$$
a_{i i}\left(P_{0}\right) y_{i}\left(P_{0}\right) \leq \sum_{Q \in M^{\prime}\left(P_{0}\right)} \sum_{j=1}^{m} b_{i j}\left(P_{0}, Q\right) y_{i}\left(P_{0}\right)+\sum_{j=1, j \neq i}^{m} c_{i j}^{1}\left(P_{0}\right) y_{i}\left(P_{0}\right)+f_{i}\left(P_{0}\right),
$$

or equivalently

$$
d_{i i}\left(P_{0}\right) y_{i}\left(P_{0}\right) \leq f_{i}\left(P_{0}\right) .
$$

From this inequality, we deduce that

$$
\max _{P \in \bar{\omega}_{h}} \mathbf{Y}(P)=y_{i}\left(P_{0}\right) \leq \frac{f_{i}\left(P_{0}\right)}{d_{i i}\left(P_{0}\right)} \leq \max _{P \in \omega_{h}} \frac{f_{i}(P)}{d_{i i}(P)} \leq \max _{P \in \omega_{h}}\left(D^{-1}(P) \mathbf{F}(P)\right) .
$$

From (11) and (12), the required upper bound is obtained

$$
y_{k}(P) \leq \max _{P \in \bar{\omega}_{h}} \mathbf{Y}(P) \leq \max \left\{\max _{P \in \partial w_{h}} \boldsymbol{\mu}(P), \max _{P \in \omega_{h}}\left(D^{-1}(P) \mathbf{F}(P)\right)\right\}, \quad k=1, \ldots, m .
$$

Finally, it is obvious that if the solution satisfies the two-side estimate (8) then, according to definition 1 , the finite difference scheme is monotone. $\nabla$

Following the proof of the previous theorem, a stability estimate in the maximum norm is obtained. In the next theorem we summarize this result.

Theorem 2. If the coefficients of problem (5)-(6) satisfy the positivity conditions (7), then the following stability estimate in the maximum norm holds

$$
\|\mathbf{Y}\|_{\bar{\omega}_{h}} \leq \max \left\{\|\boldsymbol{\mu}\|_{\partial \omega_{h}},\left\|D^{-1} \mathbf{F}\right\|_{\omega_{h}}\right\}
$$


ing

Proof. The grid function $|\mathbf{Y}(P)|$ can attain its maximum at a boundary point, yield-

$$
\max _{P \in \bar{\omega}_{h}}|\mathbf{Y}(P)|=\max _{P \in \partial \omega_{h}}|\boldsymbol{\mu}(P)|,
$$

or at an interior point $P_{0} \in \omega_{h}$, i.e., it exists $i \in\{1,2, \ldots, m\}$ such that

$$
\max _{P \in \bar{\omega}_{h}}|\mathbf{Y}(P)|=\left|y_{i}\left(P_{0}\right)\right| .
$$

In this latter case, we have the following inequality

$$
a_{i i}\left(P_{0}\right)\left|y_{i}\left(P_{0}\right)\right| \leq \sum_{Q \in M^{\prime}\left(P_{0}\right)} \sum_{j=1}^{m} b_{i j}\left(P_{0}, Q\right)\left|y_{i}\left(P_{0}\right)\right|+\sum_{j=1, j \neq i}^{m} c_{i j}^{1}\left(P_{0}\right)\left|y_{i}\left(P_{0}\right)\right|+\left|f_{i}\left(P_{0}\right)\right|,
$$

or equivalently

$$
d_{i i}\left|y_{i}\left(P_{0}\right)\right| \leq\left|f_{i}\left(P_{0}\right)\right|
$$

deducing that

$$
\max _{P \in \bar{\omega}_{h}}|\mathbf{Y}(P)|=\left|y_{i}\left(P_{0}\right)\right| \leq \frac{\left|f_{i}\left(P_{0}\right)\right|}{d_{i i}\left(P_{0}\right)} \leq \max _{P \in \omega_{h}}\left|\frac{f_{i}(P)}{d_{i i}(P)}\right| \leq \max _{P \in \omega_{h}}\left|D^{-1}(P) \mathbf{F}(P)\right| .
$$

From (14) and (15), the required estimate is obtained. $\square$

Remark 1. It is easy to show that under the fulfillment of positivity conditions (7) problem (1)-(2) has a unique solution.

\section{A two-dimensional steady diffusion system}

In this section we consider a finite difference scheme on uniform grids for a two-dimensional weakly coupled steady problem without convection. Let $\Omega$ be a rectangular domain of length $l_{1}$ in the $x_{1}$-direction and $l_{2}$ in the $x_{2}$-direction, $\Omega=\left\{0<x_{1}<l_{1}, 0<x_{2}<l_{2}\right\}$. We consider on the domain $\Omega$ a system of $m$ weakly coupled reaction-diffusion equations

$$
\left(\begin{array}{ccc}
-\Delta_{1} & 0 & \\
0 & \ddots & 0 \\
& 0 & -\Delta_{m}
\end{array}\right)\left(\begin{array}{c}
u_{1} \\
\vdots \\
u_{m}
\end{array}\right)=\left(\begin{array}{ccc}
c_{11} & \ldots & c_{1 m} \\
\vdots & & \vdots \\
c_{m 1} & \ldots & c_{m m}
\end{array}\right)\left(\begin{array}{c}
u_{1} \\
\vdots \\
u_{m}
\end{array}\right)+\left(\begin{array}{c}
f_{1}(\mathbf{x}) \\
\vdots \\
f_{m}(\mathbf{x})
\end{array}\right),
$$

with Dirichlet boundary conditions

$$
u_{k}(\mathbf{x})=\mu_{k}(\mathbf{x}), \quad k=1, \ldots, m, \quad \mathbf{x} \in \partial \Omega .
$$

This problem can be written in a matrix form as

$$
\begin{aligned}
\mathcal{L} \mathbf{U} & =\mathcal{C} \mathbf{U}+\mathbf{F}(\mathbf{x}), \quad \mathbf{x} \in \Omega, \\
\mathbf{U}(\mathbf{x}) & =\boldsymbol{\mu}(\mathbf{x}), \quad \mathbf{x} \in \partial \Omega
\end{aligned}
$$

where

$\mathbf{U}(\mathbf{x})=\left(u_{1}(\mathbf{x}), u_{2}(\mathbf{x}), \ldots, u_{m}(\mathbf{x})\right)^{T}$ is the unknown vector function, $\mathcal{C}=\left(c_{i j}\right)_{m \times m}$ is a matrix of constant coefficients,

$\mathbf{F}(\mathbf{x})=\left(f_{1}(\mathbf{x}), f_{2}(\mathbf{x}), \ldots, f_{m}(\mathbf{x})\right)^{T}$ and $\boldsymbol{\mu}(\mathbf{x})=\left(\mu_{1}(\mathbf{x}), \mu_{2}(\mathbf{x}), \ldots, \mu_{m}(\mathbf{x})\right)^{T}$ are the righthand side and boundary vector functions respectively, 
and $\mathcal{L}=-\operatorname{diag}\left(\Delta_{1}, \Delta_{2}, \ldots, \Delta_{m}\right)$ is the vector Laplacian operator in the Cartesian coordinate system, with

$$
\Delta_{k} u_{k}=\frac{\partial^{2} u_{k}}{\partial x_{1}^{2}}+\frac{\partial^{2} u_{k}}{\partial x_{2}^{2}}, \quad k=1, \ldots, m .
$$

We assume that the solution of the problem (16)-(17) exists and is unique, and all coefficients, that are included in the equation (16), and the desired function have continuous bounded derivatives, which are necessary in the course of the expansion of order. One interesting solvability approach for the elliptic system (16)-(17) is presented in the work [19] (see Theorem 3).

Let $N_{1}$ and $N_{2}$ be positive integers and let $h_{1}=l_{1} / N_{1}$ and $h_{2}=l_{2} / N_{2}$ be the space discretization parameters. We introduce the uniform grid $\bar{\omega}_{h}=\omega_{h} \cup \partial \omega_{h}$ as

$$
\bar{\omega}_{h}=\left\{\mathbf{x}_{i_{1} i_{2}}=\left(i_{1} h_{1}, i_{2} h_{2}\right), i_{\alpha}=0,1, \ldots, N_{\alpha}, \alpha=1,2\right\},
$$

where $\omega_{h}$ and $\partial \omega_{h}$ are the set of interior and boundary grid nodes of $\bar{\omega}_{h}$ respectively. On this grid, we approximate the solution of problem (16)-(17) by the finite difference scheme

$$
\begin{aligned}
& \left(\begin{array}{ccc}
-\Delta_{1 h} & 0 & \\
0 & \ddots & 0 \\
& 0 & -\Delta_{m h}
\end{array}\right)\left(\begin{array}{c}
y_{1} \\
\vdots \\
y_{m}
\end{array}\right)=\left(\begin{array}{ccc}
c_{11} & \ldots & c_{1 m} \\
\vdots & & \vdots \\
c_{m 1} & \ldots & c_{m m}
\end{array}\right)\left(\begin{array}{c}
y_{1} \\
\vdots \\
y_{m}
\end{array}\right)+\left(\begin{array}{c}
f_{1}(\mathbf{x}) \\
\vdots \\
f_{m}(\mathbf{x})
\end{array}\right), \\
& \mathbf{x} \in \omega_{h}, \\
& y_{k}(\mathbf{x})=\mu_{k}(\mathbf{x}), k=1, \ldots, m, \quad \mathbf{x} \in \partial \omega_{h},
\end{aligned}
$$

where $\Delta_{k h}$ is the standard discretization of the Laplacian operator, $\Delta_{k h} y_{k}=\left(y_{k}\right)_{\bar{x}_{1} x_{1}}+$ $\left(y_{k}\right)_{\bar{x}_{2} x_{2}}$. This scheme can be rewritten in the canonical form (5)-(6) as

$$
\begin{aligned}
A_{1} \mathbf{Y}_{i_{1} i_{2}}= & B_{1} \mathbf{Y}_{i_{1}-1 i_{2}}+B_{2} \mathbf{Y}_{i_{1} i_{2}-1}+B_{3} \mathbf{Y}_{i_{1}+1 i_{2}}+B_{4} \mathbf{Y}_{i_{1} i_{2}+1} \\
& +C_{1} \mathbf{Y}_{i_{1} i_{2}}+\mathbf{F}_{i_{1} i_{2}}, i_{\alpha}=1, \ldots, N_{\alpha}-1, \alpha=1,2, \\
\mathbf{Y}(\mathbf{x})= & \boldsymbol{\mu}(\mathbf{x}), \quad \mathbf{x} \in \partial \omega_{h},
\end{aligned}
$$

with $A_{1}$ a diagonal matrix of order $m$ with entries $\left(A_{1}\right)_{i i}=2 h_{1}^{-2}+2 h_{2}^{-2}-c_{i i}, B_{1}=B_{3}=$ $h_{1}^{-2} I_{m}$ and $B_{2}=B_{4}=h_{2}^{-2} I_{m}$. Here, $I_{m}$ denotes the identity matrix of order $m$. For the entries of $C_{1}$ we have

$$
\left(C_{1}\right)_{i j}= \begin{cases}c_{i j}, & \text { if } i \neq j, \\ 0, & \text { if } i=j .\end{cases}
$$

We observe that if the coefficients of matrix $C$ satisfy the inequalities

$$
c_{k k}<0, \quad c_{k l} \geq 0, k \neq l, \quad \sum_{l=1}^{m} c_{k l}<0, \quad k, l=1, \ldots, m,
$$

then finite difference scheme (21) satisfies the positive conditions (7), and the following results arise from Theorems 1 and 2 .

Theorem 3. If the coefficients of matrix $C$ in problem (16) satisfy the conditions (25), then finite difference scheme (21) is monotone and we have the following two-side estimates for its solution

$$
m_{1} \leq y_{k}(\mathbf{x}) \leq m_{2}, \quad \mathbf{x} \in \bar{\omega}_{h}, \quad k=1, \ldots, m
$$


where

$$
\begin{aligned}
& m_{1}=\min \left\{\min _{\mathbf{x} \in \partial w_{h}} \boldsymbol{\mu}(\mathbf{x}), \min _{\mathbf{x} \in \omega_{h}}\left(D^{-1} \mathbf{F}(\mathbf{x})\right)\right\}, \\
& m_{2}=\max \left\{\max _{\mathbf{x} \in \partial w_{h}} \boldsymbol{\mu}(\mathbf{x}), \max _{\mathbf{x} \in \omega_{h}}\left(D^{-1} \mathbf{F}(\mathbf{x})\right)\right\},
\end{aligned}
$$

with $D$ the diagonal matrix with positive entries $d_{k k}=-\sum_{l=1}^{m} c_{k l}$.

Theorem 4. If the coefficients of matrix $C$ in problem (16) satisfy the conditions (25), the following estimate holds

$$
\|\mathbf{Y}\|_{\bar{\omega}_{h}} \leq \max \left\{\|\boldsymbol{\mu}\|_{\partial \omega_{h}},\left\|D^{-1} \mathbf{F}\right\|_{\omega_{h}}\right\}
$$

\section{Two-dimensional steady convection-diffusion-reaction system}

In this section we construct a monotone finite difference scheme on uniform grids for a two-dimensional steady weakly coupled convection-diffusion-reaction system. For an overview of schemes for convection-diffusion problems see the monographs $[12,17]$. Let $\Omega$ be a rectangular domain, $\Omega=\left\{\left(x_{1}, x_{2}\right), 0<x_{1}<l_{1}, 0<x_{2}<l_{2}\right\}$ with boundary $\partial \Omega$. We consider on the domain $\Omega$ a system of $m$ coupled convection-diffusion equations

$$
\begin{aligned}
-\mathcal{L} \mathbf{U} & =\mathcal{C} \mathbf{U}+\mathbf{F}(\mathbf{x}), \quad \mathbf{x} \in \Omega, \\
\mathbf{U}(\mathbf{x}) & =\boldsymbol{\mu}(\mathbf{x}), \quad \mathbf{x} \in \partial \Omega .
\end{aligned}
$$

Here $\mathbf{U}=\mathbf{U}(\mathbf{x})$ is the unknown vector function that is sought, while $\mathcal{L}=\operatorname{diag}\left(\mathcal{L}_{1}, \mathcal{L}_{2}, \ldots, \mathcal{L}_{m}\right)$, $\mathcal{L}_{k}=\sum_{\alpha=1}^{2}\left(\mathcal{L}_{k \alpha}^{d}+\mathcal{L}_{k \alpha}^{c}\right), k=1, \ldots, m$, with

$$
\mathcal{L}_{k \alpha}^{d}=\frac{\partial}{\partial x_{\alpha}}\left(k_{k \alpha}(\mathbf{x}) \frac{\partial}{\partial x_{\alpha}}\right), \quad \mathcal{L}_{k \alpha}^{c}=r_{k \alpha} \frac{\partial}{\partial x_{\alpha}},
$$

where the functions $k_{k \alpha}$ fulfill the property $0<k_{\min } \leq k_{k \alpha}(\mathbf{x}) \leq k_{\max }, k=1, \ldots, m, \alpha=$ 1,2. As in Section $3, \mathcal{C}=\left(c_{i j}\right)_{m \times m}$ is a matrix of constant coefficients, and $\mathbf{F}(\mathbf{x})$ and $\boldsymbol{\mu}(\mathbf{x})$ are the right-hand side and boundary vector functions respectively.

On the grid $\bar{\omega}_{h}(20)$, we consider the second order finite difference scheme [14]

$$
\begin{aligned}
-\sum_{\alpha=1}^{2} \Lambda_{k \alpha} y_{k}(\mathbf{x}) & =\sum_{l=1}^{m} c_{k l} y_{l}(\mathbf{x})+f_{k}(\mathbf{x}), \quad x \in \omega_{h}, \quad k=1, \ldots, m, \\
y_{k}(\mathbf{x}) & =\mu_{k}(\mathbf{x}), \quad \mathbf{x} \in \partial \omega_{h}, \quad k=1, \ldots, m,
\end{aligned}
$$

where $\Lambda_{k \alpha} y_{k}=\varkappa_{k \alpha} \Lambda_{k \alpha}^{d} y_{k}+\Lambda_{k \alpha}^{c} y_{k}$ with $\varkappa_{k \alpha}=\left(1+R_{k \alpha}\right)^{-1}, R_{k \alpha}=0.5 h_{\alpha}\left|r_{k \alpha}\right| / k_{k \alpha}$, and

$$
\begin{aligned}
& \Lambda_{k \alpha}^{d} y_{k}=\left(a_{k \alpha} y_{k, \bar{x}_{\alpha}}\right)_{x_{\alpha}}, a_{k 1}=k_{k 1}\left(x_{1}-h_{1} / 2, x_{2}\right), a_{k 2}=k_{k 2}\left(x_{1}, x_{2}-h_{2} / 2\right), \\
& \Lambda_{k \alpha}^{c} y_{k}=b_{k \alpha}^{+} a_{k \alpha}^{(+1)} y_{k, x_{\alpha}}+b_{k \alpha}^{-} a_{k \alpha} y_{k, \bar{x}_{\alpha}}, \quad b_{k \alpha}^{ \pm}=\frac{r_{k \alpha}^{ \pm}}{k_{k \alpha}}, r_{k \alpha}^{ \pm}=0.5\left(r_{k \alpha} \pm\left|r_{k \alpha}\right|\right) .
\end{aligned}
$$

This scheme can be rewritten in the canonical form (5)-(6) as

$$
\begin{aligned}
& A_{1}\left(\mathbf{x}_{i_{1} i_{2}}\right) \mathbf{Y}_{i_{1} i_{2}}=B_{1}\left(\mathbf{x}_{i_{1} i_{2}}\right) \mathbf{Y}_{i_{1}-1 i_{2}}+B_{2}\left(\mathbf{x}_{i_{1} i_{2}}\right) \mathbf{Y}_{i_{1} i_{2}-1}+B_{3}\left(\mathbf{x}_{i_{1} i_{2}}\right) \mathbf{Y}_{i_{1}+1 i_{2}} \\
& +B_{4}\left(\mathbf{x}_{i_{1} i_{2}}\right) \mathbf{Y}_{i_{1} i_{2}+1}+C_{1}\left(\mathbf{x}_{i_{1} i_{2}}\right) \mathbf{Y}_{i_{1} i_{2}}+\mathbf{F}_{i_{1} i_{2}}, i_{\alpha}=1, \ldots, N_{\alpha}-1, \alpha=1,2 \\
& \mathbf{Y}(\mathbf{x})=\boldsymbol{\mu}(\mathbf{x}), \quad \mathbf{x} \in \partial \omega_{h}
\end{aligned}
$$


where $B_{j}\left(\mathbf{x}_{i_{1} i_{2}}\right)=\operatorname{diag}\left(b_{11}^{j}\left(\mathbf{x}_{i_{1} i_{2}}\right), \ldots, b_{m m}^{j}\left(\mathbf{x}_{i_{1} i_{2}}\right)\right), j=1, \ldots, 4$ with entries for $k=$ $1, \ldots, m$

$$
\begin{aligned}
b_{k k}^{1}\left(\mathbf{x}_{i_{1} i_{2}}\right) & =\frac{a_{k 1, i_{1}-1 / 2 i_{2}}}{h_{1}^{2}}\left(\varkappa_{k 1, i_{1} i_{2}}-h_{1} b_{k 1, i_{1} i_{2}}^{-}\right), \\
b_{k k}^{2}\left(\mathbf{x}_{i_{1} i_{2}}\right) & =\frac{a_{k 2, i_{1} i_{2}-1 / 2}}{h_{2}^{2}}\left(\varkappa_{k 2, i_{1} i_{2}}-h_{2} b_{k 2, i_{1} i_{2}}^{-}\right), \\
b_{k k}^{3}\left(\mathbf{x}_{i_{1} i_{2}}\right) & =\frac{a_{k 1, i_{1}+1 / 2 i_{2}}}{h_{1}^{2}}\left(\varkappa_{k 1, i_{1} i_{2}}+h_{1} b_{k 1, i_{1} i_{2}}^{+}\right), \\
b_{k k}^{4}\left(\mathbf{x}_{i_{1} i_{2}}\right) & =\frac{a_{k 2, i_{1} i_{2}+1 / 2}}{h_{2}^{2}}\left(\varkappa_{k 2, i_{1} i_{2}}+h_{2} b_{k 2, i_{1} i_{2}}^{+}\right),
\end{aligned}
$$

$A_{1}\left(\mathbf{x}_{i_{1} i_{2}}\right)$ is a diagonal matrix with diagonal entries $a_{k k}^{1}\left(\mathbf{x}_{i_{1} i_{2}}\right)=b_{k k}^{1}\left(\mathbf{x}_{i_{1} i_{2}}\right)+b_{k k}^{2}\left(\mathbf{x}_{i_{1} i_{2}}\right)+$ $b_{k k}^{3}\left(\mathbf{x}_{i_{1} i_{2}}\right)+b_{k k}^{4}\left(\mathbf{x}_{i_{1} i_{2}}\right)-c_{k k}$ and $C_{1}\left(\mathbf{x}_{i_{1} i_{2}}\right)=\left(c_{k l}^{1}\right)_{m \times m}$ is a matrix such that $c_{k l}^{1}=c_{k l}$ if $k \neq l$, and zero in other case.

From the above coefficients, it is easy to see that if the entries of matrix $C$ satisfy the inequalities

$$
c_{k k}<0, \quad c_{k l} \geq 0, k \neq l, \quad \sum_{l=1}^{m} c_{k l}<0, \quad k, l=1, \ldots, m,
$$

then finite difference scheme (32)-(33) satisfies the positive conditions (7), and therefore applying Theorem 1 such scheme is monotone. Moreover, from Theorem 2 we have a priori estimate of the solution, which is summarized in the next result.

Theorem 5. If the coefficients of matrix $C$ in problem (29) satisfy the conditions (34), the following estimate holds

$$
\|\mathbf{Y}\|_{\bar{\omega}_{h}} \leq \max \left\{\|\boldsymbol{\mu}\|_{\partial \omega_{h}},\left\|D^{-1} \mathbf{F}\right\|_{\omega_{h}}\right\}
$$

where $D$ is a diagonal matrix with entries $d_{k k}=-\sum_{l=1}^{m} c_{k l}$.

\section{A weakly coupled system of two-dimensional quasi- linear parabolic equations}

Let $\Omega$ be the rectangular domain $\Omega=\left\{\left(x_{1}, x_{2}\right), 0<x_{1}<l_{1}, 0<x_{2}<l_{2}\right\}$ and $\partial \Omega$ its boundary. For a fixed positive number $T>0$, we consider the weakly coupled system of $m$ quasi-linear parabolic equations

$$
\frac{\partial \mathbf{U}}{\partial t}=\mathcal{L} \mathbf{U}+\mathcal{C} \mathbf{U}+\mathbf{F}_{1}(\mathbf{x}, t), \quad \mathbf{x} \in \Omega, \quad 0<t \leq T,
$$

with Dirichlet boundary conditions

$$
\mathbf{U}(\mathbf{x}, t)=\boldsymbol{\mu}(\mathbf{x}, t), \quad \mathbf{x} \in \partial \Omega, \quad 0<t \leq T,
$$

and initial condition

$$
\mathbf{U}(\mathbf{x}, 0)=\mathbf{U}_{0}(\mathbf{x}), \quad \mathbf{x} \in \Omega \cup \partial \Omega,
$$

where $\mathbf{U}(\mathbf{x}, t)=\left(u_{1}(\mathbf{x}, t), u_{2}(\mathbf{x}, t), \ldots, u_{m}(\mathbf{x}, t)\right)$ is the sought vector grid function, and $\mathcal{L}$ is a diagonal operator $\mathcal{L}=\operatorname{diag}\left(\mathcal{L}_{1}, \mathcal{L}_{2}, \ldots, \mathcal{L}_{m}\right)$,

$$
\begin{aligned}
\mathcal{L}_{k} u_{k} & =\sum_{\alpha=1}^{2} \frac{\partial}{\partial x_{\alpha}}\left(k_{k \alpha}(\mathbf{U}) \frac{\partial u_{k}}{\partial x_{\alpha}}\right), \quad k=1, \ldots, m, \\
k_{k \alpha}(\mathbf{U}) & =k_{k \alpha}\left(u_{1}, u_{2}, \ldots, u_{m}\right) .
\end{aligned}
$$


We suppose that the input data is non-negative $\mathbf{F}_{1}(\mathbf{x}, t) \geq 0, \boldsymbol{\mu}(\mathbf{x}, t) \geq 0, \mathbf{U}_{0}(\mathbf{x}) \geq 0$ and each function $k_{k \alpha}(\mathbf{U}), k=1, \ldots, m, \alpha=1,2$, fulfills the property

$$
0<k_{\min } \leq k_{k \alpha}(\mathbf{U}) \leq k_{\max }, \quad \forall \mathbf{U}=\left(u_{k}\right)_{k=1}^{m}, u_{k} \in\left[\bar{m}_{1}, \bar{m}_{2}\right]
$$

where $\bar{m}_{1}$ and $\bar{m}_{2}$ are two constants such that

$$
\begin{aligned}
\bar{m}_{1} & =e^{-T c} \min \left\{\min _{\mathbf{x} \in \partial \Omega, t \in[0, T]} \boldsymbol{\mu}(\mathbf{x}, t), \min _{\mathbf{x} \in \bar{\Omega}} \mathbf{U}_{0}(\mathbf{x})+T \min _{\mathbf{x} \in \bar{\Omega}, t \in[0, T]} \mathbf{F}_{1}(\mathbf{x}, t)\right\}, \\
\bar{m}_{2} & =\max \left\{\max _{\mathbf{x} \in \partial \Omega, t \in[0, T]} \mu(\mathbf{x}, t), \max _{\mathbf{x} \in \bar{\Omega}} \mathbf{U}_{0}(\mathbf{x})\right\}+T \max _{\mathbf{x} \in \bar{\Omega}, t \in[0, T]} \mathbf{F}_{1}(\mathbf{x}, t), \\
c & =\max _{1 \leq i \leq m}\left(-\sum_{j=1}^{m} c_{i j}\right)>0,
\end{aligned}
$$

with $\bar{Q}_{T}=\bar{\Omega} \times[0, T]$. Again, as in previous sections, $\mathcal{C}=\left(c_{i j}\right)_{m \times m}$ is a matrix of constant coefficients satisfying the conditions

$$
c_{k k}<0, \quad c_{k l} \geq 0, k \neq l, \quad \sum_{l=1}^{m} c_{k l}<0, \quad k, l=1, \ldots, m .
$$

Let $N_{0}$ be positive integers and let $\tau=T / N_{0}$ be the time discretization parameters. Then, we introduce the uniform grids $\bar{\omega}_{h \tau}=\bar{\omega}_{h} \times \bar{\omega}_{\tau}$, with $\bar{\omega}_{h}=\omega_{h} \cup \partial \omega_{h}(20)$ and

$$
\bar{\omega}_{\tau}=\left\{t_{n}=n \tau, n=0,1, \ldots, N_{0}\right\} .
$$

On the grid $\bar{\omega}_{h \tau}$, we approximate the initial-boundary value problem (36)-(38) by a standard first order backward implicit scheme

$$
\begin{aligned}
\mathbf{Y}_{t}^{n} & =L_{h} \mathbf{Y}^{n+1}+C \mathbf{Y}^{n+1}+\mathbf{F}_{1}^{n+1}(\mathbf{x}), \quad \mathbf{x} \in \omega_{h}, \quad n=0, \ldots, N_{0}-1, \\
\mathbf{Y}^{0} & =\mathbf{U}_{0}(\mathbf{x}), \quad \mathbf{x} \in \bar{\omega}_{h}, \\
\mathbf{Y}^{n+1} & =\boldsymbol{\mu}\left(\mathbf{x}, t_{n+1}\right), \quad \mathbf{x} \in \partial \omega_{h}, \quad n=0,1, \ldots, N_{0}-1,
\end{aligned}
$$

where $\mathbf{Y}(\mathbf{x}, t)=\left(y_{1}(\mathbf{x}, t), y_{2}(\mathbf{x}, t), \ldots, y_{m}(\mathbf{x}, t)\right)^{T}$ is the vector approximation grid-function, and $\mathbf{Y}_{t}^{n}(\mathbf{x}, t)=\left(y_{1 t}^{n}(\mathbf{x}, t), y_{2 t}^{n}(\mathbf{x}, t), \ldots, y_{m t}^{n}(\mathbf{x}, t)\right)^{T}$, with

$$
y_{k t}^{n}(\mathbf{x}, t)=\frac{y_{k}(\mathbf{x}, t+\tau)-y_{k}(\mathbf{x}, t)}{\tau}, \quad k=1, \ldots, m .
$$

Discrete operator $L_{h}$ is a diagonal operator $L_{h}=\operatorname{diag}\left(L_{1 h}, L_{2 h}, \ldots, L_{m h}\right)$, where

$$
L_{k h} y_{k, i_{\alpha}}^{n+1}=\sum_{\alpha=1}^{2}\left(a_{k \alpha}\left(\mathbf{Y}_{i_{\alpha}}^{n}\right) y_{k, \bar{x}_{\alpha}}^{n+1}\right)_{x_{\alpha}, i_{\alpha}}, \quad k=1, \ldots, m, \quad \alpha=1,2,
$$

with

$$
\begin{aligned}
\left(a_{k \alpha}\left(\mathbf{Y}_{i_{\alpha}}^{n}\right) y_{k, \bar{x}_{\alpha}}^{n+1}\right)_{x_{\alpha}, i_{\alpha}} & =a_{k \alpha, i_{\alpha}+1} \frac{y_{k, i_{\alpha}+1}^{n+1}-y_{k, i_{\alpha}}^{n+1}}{h_{\alpha}^{2}}-a_{k \alpha, i_{\alpha}} \frac{y_{k, i_{\alpha}}^{n+1}-y_{k, i_{\alpha}-1}^{n+1}}{h_{\alpha}^{2}} \\
a_{k \alpha, i_{\alpha}} & =\frac{1}{2}\left(k_{k \alpha}\left(\mathbf{Y}_{i_{\alpha}-1}^{n}\right)+k_{k \alpha}\left(\mathbf{Y}_{i_{\alpha}}^{n}\right)\right) .
\end{aligned}
$$

Notice that we have constructed a finite difference scheme of second-order of approximation in space. This can be easily deduced from the following lemma 
Lemma 1. The following approximation holds

$$
\psi_{k}=\left(a(\mathbf{U})\left(u_{k}\right)_{\bar{x}}\right)_{x}-\frac{\partial}{\partial x}\left(k(\mathbf{U}) \frac{\partial u_{k}}{\partial x}\right)=O\left(h^{2}\right),
$$

where

$$
a(\mathbf{U})=\frac{k(\mathbf{U})_{i}+k(\mathbf{U})_{i-1}}{2} .
$$

Proof. By using the formula of summation by parts $(f g)_{x}=f_{(0.5)} g_{x}+f_{x} g_{(0.5)}$, where $v_{(0.5)}=\left(v+v_{+}\right) / 2, v_{x}=\left(v_{+}-v\right) / h$ the approximation error $\psi$ can be split up as $\psi_{k}=\psi_{k 1}+\psi_{k 2}$, where

$$
\begin{aligned}
& \psi_{k 1}=a(\mathbf{U})_{x}\left(\left(u_{k}\right)_{\bar{x}}\right)_{(0.5)}-\frac{\partial}{\partial x} k(\mathbf{U}) \frac{\partial u_{k}}{\partial x}, \\
& \psi_{k 2}=a(\mathbf{U})_{(0.5)}\left(u_{k}\right)_{\bar{x} x}-k(\mathbf{U}) \frac{\partial^{2} u_{k}}{\partial x^{2}} .
\end{aligned}
$$

Taking into account that $a(\mathbf{U})_{(0.5)}=k(\mathbf{U})+O\left(h^{2}\right),\left(\left(u_{k}\right)_{\bar{x}}\right)_{(0.5)}=\frac{\partial u_{k}}{\partial x}+O\left(h^{2}\right)$ and $a(\mathbf{U})_{x}=\frac{\partial}{\partial x} k(\mathbf{U})+O\left(h^{2}\right)$, we obtain the required result. $\otimes$

Scheme (40)-(42) can be rewritten in the canonical form (5)-(6) as

$$
\begin{aligned}
A_{1} \mathbf{Y}_{i_{1} i_{2}}^{n+1}= & B_{1} \mathbf{Y}_{i_{1}-1 i_{2}}^{n+1}+B_{2} \mathbf{Y}_{i_{1} i_{2}-1}^{n+1}+B_{3} \mathbf{Y}_{i_{1}+i_{2}}^{n+1}+B_{4} \mathbf{Y}_{i_{1} i_{2}+1}^{n+1} \\
& +C_{1} \mathbf{Y}_{i_{1} i_{2}}^{n+1}+\mathbf{F}_{i_{1} i_{2}}^{n+1}, i_{\alpha}=1, \ldots, N_{\alpha}-1, \alpha=1,2, \\
\mathbf{Y}^{n+1}(\mathbf{x})= & \boldsymbol{\mu}^{n+1}(\mathbf{x}), \quad \mathbf{x} \in \partial \omega_{h},
\end{aligned}
$$

with $A_{1}$ a diagonal matrix of order $m$ with entries

$$
a_{k k}^{1}=1+\frac{\tau}{h_{1}^{2}}\left(a_{k 1, i_{1}+1 i_{2}}^{n}+a_{k 1, i_{1} i_{2}}^{n}\right)+\frac{\tau}{h_{2}^{2}}\left(a_{k 2, i_{1} i_{2}+1}^{n}+a_{k 2, i_{1} i_{2}}^{n}\right)-\tau c_{k k} .
$$

Matrices $B_{j}, j=1, \ldots, 4$, are diagonal matrices $B_{j}=\operatorname{diag}\left(b_{11}^{j}, b_{22}^{j} \ldots, b_{m m}^{j}\right)$, with entries for $k=1, \ldots, m$

$$
b_{k k}^{1}=\frac{\tau}{h_{1}^{2}} a_{k 1, i_{1} i_{2}}^{n}, \quad b_{k k}^{2}=\frac{\tau}{h_{2}^{2}} a_{k 2, i_{1} i_{2}}^{n}, \quad b_{k k}^{3}=\frac{\tau}{h_{1}^{2}} a_{k 1, i_{1}+1 i_{2}}^{n}, \quad b_{k k}^{4}=\frac{\tau}{h_{2}^{2}} a_{k 2, i_{1} i_{2}+1}^{n},
$$

$\mathbf{F}_{i_{1} i_{2}}^{n+1}=\tau \mathbf{F}_{1, i_{1} i_{2}}^{n+1}+\mathbf{Y}_{i_{1} i_{2}}^{n}$ and $C_{1}=\left(c_{k l}^{1}\right)_{m \times m}$ is a matrix such that $c_{k l}^{1}=\tau c_{k l}$ if $k \neq l$, and zero in other case.

From the above coefficients, we obtain the following theorem about monotonicity of FDS (40)-(42).

Theorem 6. For the solution of the difference scheme (40)-(42) the two-side estimate

$$
\tilde{m}_{1} \leq y_{k}^{n+1}(\mathbf{x}) \leq \tilde{m}_{2}, \quad \mathbf{x} \in \bar{\omega}_{h}, \quad k=1,2, \ldots, m,
$$

holds, where

$$
\begin{aligned}
\tilde{m}_{1} & =e^{-T c} \min \left\{\min _{\substack{0 \leq k \leq N_{0}-1 \\
\mathbf{x} \in \partial \bar{\omega}_{h}}} \boldsymbol{\mu}^{k+1}(\mathbf{x}), \min _{\mathbf{x} \in \bar{\omega}_{h}} \mathbf{U}_{0}(\mathbf{x})+T \min _{\substack{0 \leq k \leq N_{0}-1 \\
\mathbf{x} \in \bar{\omega}_{h}}} \mathbf{F}_{1}^{k+1}(\mathbf{x})\right\} \geq \bar{m}_{1}, \\
\tilde{m}_{2} & =\max \left\{\max _{\substack{0 \leq k \leq N_{0}-1 \\
\mathbf{x} \in \partial \omega_{h}}} \mu^{k+1}(\mathbf{x}), \max _{\mathbf{x} \in \bar{\omega}_{h}} \mathbf{U}_{0}(\mathbf{x})\right\}+T \max _{\substack{0 \leq k \leq N_{0}-1 \\
\mathbf{x} \in \bar{\omega}_{h}}} \mathbf{F}_{1}^{k+1}(\mathbf{x}) \leq \bar{m}_{2}, \\
c & =\max _{1 \leq i \leq m}\left(-\sum_{j=1}^{m} c_{i j}\right)>0,
\end{aligned}
$$

and therefore the finite difference scheme is monotone. 
Proof. We prove the lower bound of estimate (45). In a similar way, the upper bound can be proved. The minimum of the vector grid function $\mathbf{Y}^{n+1}(\mathbf{x}), \mathbf{x}=\left(x_{1}, x_{2}\right) \in \bar{\omega}_{h}$ in sense of notation (3) can be reached at some function $y_{i}^{n+1}(\mathbf{x}), i \in\{1,2, \ldots, m\}$, i.e.

$$
\min _{\mathbf{x} \in \bar{\omega}_{h}} \mathbf{Y}^{n+1}(\mathbf{x})=\min _{\mathbf{x} \in \bar{\omega}_{h}} y_{i}^{n+1}(\mathbf{x}), \quad i \in\{1,2, \ldots, m\} .
$$

Then from Theorem 1, we have

$$
\begin{aligned}
\min _{\mathbf{x} \in \bar{\omega}_{h}} y_{i}^{n+1}(\mathbf{x}) & \geq \min \left\{\min _{\mathbf{x} \in \partial \omega_{h}} \mu_{i}^{n+1}(\mathbf{x}), \frac{1}{d_{i i}}\left(\min _{\mathbf{x} \in \bar{\omega}_{h}} y_{i}^{n}(\mathbf{x})+\tau \min _{\mathbf{x} \in \omega_{h}} f_{i}^{n+1}(\mathbf{x})\right)\right\}, \\
& \geq \frac{1}{d_{i i}} \min \left\{\min _{\mathbf{x} \in \gamma_{h}} \mu_{i}^{n+1}(\mathbf{x}), \min _{\mathbf{x} \in \bar{\omega}_{h}} y_{i}^{n}(\mathbf{x})+\tau \min _{\mathbf{x} \in \omega_{h}} f_{i}^{n+1}(\mathbf{x})\right\} .
\end{aligned}
$$

It's easy to see that $y_{i}^{n+1}(\mathbf{x}) \geq 0$. So from (46) by recursive process, we obtain

$$
\begin{aligned}
& \min _{\mathbf{x} \in \bar{\omega}_{h}} y_{i}^{n+1}(\mathbf{x}) \geq \\
& \geq \frac{1}{\left(d_{i i}\right)^{n+1}} \min \left\{\min _{\substack{0 \leq k \leq N_{0}-1 \\
\mathbf{x} \in \partial \omega_{h}}} \mu_{i}^{k+1}(\mathbf{x}), \min _{\mathbf{x} \in \omega_{h}} u_{i 0}(\mathbf{x})+T \min _{\substack{0 \leq k \leq N_{0}-1 \\
\mathbf{x} \in \partial \omega_{h}}} f_{i}^{k+1}(\mathbf{x})\right\} .
\end{aligned}
$$

Using estimate $e^{-c T} \leq \frac{1}{\left(d_{i i}\right)^{n+1}} \leq \frac{1}{d_{i i}} \leq 1, c=\max _{1 \leq i \leq m}\left(-\sum_{j=1}^{m} c_{i j}\right)>0$ we obtain (45). The theorem is proved. $\otimes$

Remark 2. By the similar way it is possible to prove estimates for solution of finite difference scheme (40)-(42) in cases: $\boldsymbol{\mu}(\mathbf{x}, t) \leq 0, \mathbf{F}_{1}(\mathbf{x}, t) \leq 0 ; \boldsymbol{\mu}(\mathbf{x}, t) \geq 0, \mathbf{F}_{1}(\mathbf{x}, t) \leq 0$ and $\boldsymbol{\mu}(\mathbf{x}, t) \leq 0, \mathbf{F}_{1}(\mathbf{x}, t) \geq 0$.

Theorem 7. If the coefficients of matrix $C$ in problem (36) satisfy the conditions (39), the following estimate holds

$$
\left\|\mathbf{Y}^{n+1}\right\|_{\bar{\omega}_{h}} \leq \max \left\{\max _{0 \leq k \leq N_{0}-1}\left\|\boldsymbol{\mu}^{k+1}\right\|_{\partial \omega_{h}},\left\|\mathbf{u}_{0}\right\|_{\bar{\omega}_{h}}\right\}+T \max _{0 \leq k \leq N_{0}-1}\left\|\mathbf{F}_{1}^{k+1}\right\|_{\omega_{h}} .
$$

Proof. From Theorem 2, we deduce

$$
\left\|\mathbf{Y}^{n+1}\right\|_{\bar{\omega}_{h}} \leq \max \left\{\left\|\boldsymbol{\mu}^{n+1}\right\|_{\partial \omega_{h}},\left\|D^{-1} \mathbf{F}^{n+1}\right\|_{\omega_{h}}\right\}
$$

where $D$ is a diagonal matrix with entries $d_{k k}=1-\tau \sum_{l=1}^{m} c_{k l}$. As $d_{k k} \geq 1, k=1, \ldots, m$, we have

$$
\left\|\mathbf{Y}^{n+1}\right\|_{\bar{\omega}_{h}} \leq \max \left\{\left\|\boldsymbol{\mu}^{n+1}\right\|_{\partial \omega_{h}},\left\|\mathbf{Y}^{n}\right\|_{\bar{\omega}_{h}}+\tau\left\|\mathbf{F}_{1}^{n+1}\right\|_{\omega_{h}}\right\}
$$

By recursive process, the required estimate holds. $\square$

\section{One-dimensional steady convection-diffusion-reaction system on non-uniform grids}

In this section, we study the monotonicity of a finite difference scheme on non-uniform grids for a one-dimensional steady weakly coupled convection-diffusion-reaction system. 
On the domain $\Omega=(0, L)$, we consider a system of $m$ coupled convection-diffusion equations

$$
\begin{aligned}
-\mathcal{L} \mathbf{U} & =\mathcal{C} \mathbf{U}+\mathbf{F}(x), \quad x \in(0, L), \\
\mathbf{U}(0) & =\boldsymbol{\mu}^{1}, \quad \mathbf{U}(L)=\boldsymbol{\mu}^{2},
\end{aligned}
$$

where $\mathcal{L}=\operatorname{diag}\left(\mathcal{L}_{1}, \mathcal{L}_{2}, \ldots, \mathcal{L}_{m}\right), \mathcal{L}_{k}=\mathcal{L}_{k}^{d}+\mathcal{L}_{k}^{c}, k=1, \ldots, m$, with

$$
\mathcal{L}_{k}^{d}=\frac{\mathrm{d}}{\mathrm{d} x}\left(k_{k}(x) \frac{\mathrm{d}}{\mathrm{d} x}\right), \quad \mathcal{L}_{k}^{c}=r_{k}(x) \frac{\mathrm{d}}{\mathrm{d} x},
$$

and the functions $k_{k}(x)$ fulfill the property $0<k_{\min } \leq k_{k}(x) \leq k_{\max }, k=1, \ldots, m$, with $k_{\min }$ and $k_{\max }$ two real numbers. As in previous sections $\mathcal{C}=\left(c_{i j}\right)$ is a square matrix of order $m$ of constant coefficients, $\mathbf{F}(x)$ is the right-hand side and $\boldsymbol{\mu}^{1}, \boldsymbol{\mu}^{2}$ are the boundary vectors.

Let $\bar{\omega}_{h}=\omega_{h} \cup \partial \omega_{h}$ be the non-uniform grid given by $\omega_{h}=\left\{x_{i}=x_{i-1}+h_{i}, i=\right.$ $1, \ldots, N-1\}$ and $\partial \omega_{h}=\left\{x_{0}=0, x_{N}=L\right\}$. In the sequel we will use the following notations. We denote $\hbar_{i}=0.5\left(h_{i}+h_{i+1}\right)$, and $\bar{x}_{i}=x_{i}+\tilde{h}_{i}$, with $\tilde{h}_{i}=\left(h_{i+1}-h_{i}\right) / 3$. Besides, for a independent variable $x=x_{i}$, we denote $x_{ \pm}=x_{i \pm 1}$, whereas for a grid function $v=v_{i}=v\left(x_{i}\right), v^{( \pm 1)}=v\left(x_{i \pm 1}\right)$. Moreover, we will use the standard notation,

$$
\begin{aligned}
v_{\bar{x} x, i} & =\frac{v_{x, i}-v_{\bar{x}, i}}{\hbar_{i}}, \quad v_{x, i}=\frac{v_{i+1}-v_{i}}{h_{i+1}}, \quad v_{\bar{x}, i}=\frac{v_{i}-v_{i-1}}{h_{i}}, \\
v_{\left(\beta_{k i} \beta_{k+1 i}\right)} & =\beta_{k i} v_{i+1}+\left(1-\beta_{k i}-\beta_{k+1 i}\right) v_{i}+\beta_{k+1 i} v_{i-1} .
\end{aligned}
$$

On this grid, we consider the finite difference scheme

$$
\begin{aligned}
-\Lambda_{k} y_{k} & =c_{k k} y_{k\left(\beta_{5} \beta_{6}\right)}+\sum_{l=1, l \neq k}^{m}\left(c_{k l} y_{l\left(\beta_{1} \beta_{2}\right)}\right)+\varphi_{k}, \quad k=1, \ldots, m, \\
y_{k}(0) & =\mu_{k}^{1}, \quad y_{k}(L)=\mu_{k}^{2}, \quad k=1, \ldots, m .
\end{aligned}
$$

As in the continuous case, discrete operator $\Lambda_{k}$ is split into two parts: the diffusive and the convective terms given by the following expressions $\Lambda_{k} y_{k}=\varkappa_{k} \Lambda_{k}^{D} y_{k}+\Lambda_{k}^{C} y_{k}$, where

$$
\begin{aligned}
\Lambda_{k}^{D} y_{k} & =0.5\left(k_{k\left(\beta_{1} \beta_{2}\right)} y_{k, \bar{x} x}+\left(k_{k} y_{k}\right)_{\bar{x} x}-k_{k, \bar{x} x} y_{k\left(\beta_{3} \beta_{4}\right)}\right), \\
\Lambda_{k}^{C} y_{k} & =\bar{b}_{k}^{+} a_{k}^{(+1)} y_{k, x}+\bar{b}_{k}^{-} a_{k} y_{k, \bar{x}},
\end{aligned}
$$

with $a_{k}=k_{k}(x-h / 2)$ and $\bar{b}_{k}^{ \pm}=\frac{r_{k}^{ \pm}(\bar{x})}{k_{k}(\bar{x})}, r_{k}^{+}(\bar{x})=0.5\left(r_{k}+\left|r_{k}\right|\right) \geq 0$ and $r_{k}^{-}(\bar{x})=0.5\left(r_{k}-\right.$ $\left.\left|r_{k}\right|\right) \leq 0$. Parameter $\varkappa_{k}$ is defined as $\varkappa_{k}=\frac{1}{1+R_{k}}$, where $R_{k}=\bar{b}_{k}^{+} \frac{2 h+h_{+}}{6}-\bar{b}_{k}^{-} \frac{h+2 h_{+}}{6}$, and $\varphi_{k}=f_{k}(\bar{x})$. Coefficients $\beta_{i}, i=1, \ldots, 6$ are chosen in order to obtain a monotone second-order scheme

$$
\begin{aligned}
& \beta_{1}=\frac{|\tilde{h}|+\tilde{h}}{2 h_{+}}, \quad \beta_{2}=\frac{|\tilde{h}|-\tilde{h}}{2 h}, \quad \beta_{3}=\frac{\tilde{h} k_{k \bar{x} x}-\left|\tilde{h} k_{k \bar{x} x}\right|}{2 h_{+} k_{k \bar{x} x}}, \\
& \beta_{4}=-\frac{\tilde{h} k_{k \bar{x} x}+\left|\tilde{h} k_{k \bar{x} x}\right|}{2 h k_{k \bar{x} x}}, \quad \beta_{5}=\frac{\tilde{h}-|\tilde{h}|}{2 h_{+}}, \quad \beta_{6}=-\frac{|\tilde{h}|+\tilde{h}}{2 h} .
\end{aligned}
$$

This scheme can be rewritten in the canonical form (5)-(6) as

$$
\begin{aligned}
A_{1}\left(x_{i}\right) \mathbf{Y}_{i} & =B_{1}\left(x_{i}\right) \mathbf{Y}_{i-1}+B_{2}\left(x_{i}\right) \mathbf{Y}_{i+1}+C_{1}\left(x_{i}\right) \mathbf{Y}_{i}+\mathbf{F}_{i}, i=1, \ldots, N-1, \\
\mathbf{Y}_{0} & =\boldsymbol{\mu}^{1}, \quad \mathbf{Y}_{N}=\boldsymbol{\mu}^{2},
\end{aligned}
$$


where $B_{1}\left(x_{i}\right)=\left(b_{k l}^{1}\right)_{m \times m}$ and $B_{2}\left(x_{i}\right)=\left(b_{k l}^{2}\right)_{m \times m}$ are matrices defined by the coefficients

$$
\begin{aligned}
& b_{k l}^{1}\left(x_{i}\right)=c_{k l} \beta_{2 i}, \quad b_{k l}^{2}\left(x_{i}\right)=c_{k l} \beta_{1 i}, \quad l=1, \ldots, m, l \neq k, \\
& b_{k k}^{1}\left(x_{i}\right)=\frac{\varkappa_{k i}\left(k_{k\left(\beta_{1 i} \beta_{2 i}\right)}+k_{k i-1}\right)}{2 \hbar_{i} h_{i}}-\frac{\varkappa_{k i} \beta_{4 i} k_{k \bar{x} x, i}}{2}-\frac{\bar{b}_{k}^{-} a_{k i}}{h_{i}}+c_{k k} \beta_{6 i}, \\
& b_{k k}^{2}\left(x_{i}\right)=\frac{\varkappa_{k i}\left(k_{k\left(\beta_{1 i} \beta_{2 i}\right)}+k_{k i+1}\right)}{2 \hbar_{i} h_{i+1}}-\frac{\varkappa_{k i} \beta_{3 i} k_{k \bar{x} x, i}}{2}+\frac{\bar{b}_{k}^{+} a_{k i+1}}{h_{i+1}}+c_{k k} \beta_{5 i} .
\end{aligned}
$$

$A_{1}\left(x_{i}\right)$ is a diagonal matrix with diagonal entries $a_{k k}^{1}\left(x_{i}\right)=b_{k k}^{1}\left(x_{i}\right)+b_{k k}^{2}\left(x_{i}\right)-c_{k k}$ and $C_{1}\left(x_{i}\right)=\left(c_{k l}^{1}\left(x_{i}\right)\right)_{m \times m}$ is a matrix such that $c_{k l}^{1}\left(x_{i}\right)=c_{k l}\left(1-\beta_{1 i}-\beta_{2 i}\right)$ if $k \neq l$, and zero in other case.

From the above coefficients, it is easy to see that if the entries of matrix $C$ verify the inequalities

$$
c_{k k}<0, \quad c_{k l} \geq 0, k \neq l, \quad \sum_{l=1}^{m} c_{k l}<0, \quad k, l=1, \ldots, m,
$$

then finite difference scheme (53)-(54) satisfies the positive conditions (7), and therefore applying Theorem 1 such scheme is monotone. Moreover, from Theorem 2 we have a priori estimate of the solution, which is summarized in the next result.

Theorem 8. If the coefficients of matrix $C$ in problem (48) satisfy the conditions (62), the following estimate holds

$$
\|\mathbf{Y}\|_{\bar{\omega}_{h}} \leq \max \left\{\left\|\boldsymbol{\mu}^{1}\right\|_{\partial \omega_{h}},\left\|\boldsymbol{\mu}^{2}\right\|_{\partial \omega_{h}},\left\|D^{-1} \mathbf{F}\right\|_{\omega_{h}}\right\}
$$

where $D$ is a diagonal matrix with entries $d_{k k}=-\sum_{l=1}^{m} c_{k l}$.

Remark 3. The obtained results can be generalized to semi-linear weakly coupled elliptic and parabolic system of equations with nonlinear right-hand sides [5, 13].

\section{Conclusions}

In this paper we have developed a theory of monotonicity for finite difference schemes approximating weakly coupled system of linear elliptic and quasilinear parabolic equations. Sufficient conditions of positivity of the input data are given to guarantee the property of monotonicity of the finite difference schemes. Besides, these positive conditions allow us obtain two-side estimates of the approximate solution. This theory is applied to a wide range of finite difference schemes approximating weakly coupled system of equations. In particular we consider finite differences schemes for two-dimensional weakly coupled diffusion problems, two-dimensional quasilinear parabolic systems, and one-dimensional weakly coupled elliptic systems on non-uniform grids.

Acknowledgement: F.J. Gaspar has received funding from the European Union's Horizon 2020 research and innovation programme under the Marie Sklodowska-Curie grant agreement No 705402, POROSOS.

\section{References}

[1] I. Farago and R. Horvath. Discrete maximum principle and adequate discretizations of linear parabolic problems. SIAM J. Sci. Comput., 28:2313-2336, 2006. 
[2] I. Farago, J. Karatson, and S. Korotov. Discrete maximum principles for nonlinear parabolic pde systems. IMA Journal of Numerical Analysis, 32(4):1541-1573, 2012.

[3] M.N. Koleva and L. G. Vulkov. A second-order positivity preserving numerical method for gamma equation. Appl. Math. and Comput, 220:722-734, 2013.

[4] D. Malafei. Economical monotone difference schemes for multidimensional convection-diffusion problems on non-uniform grids. Doklady NAS Belarus, 44(4):2125, In Russian, 2000.

[5] S. A. Manaa. Some numerical methods for schnackenberg model. International Journal of Engineering Inventions, 2(2):71-78, 2013.

[6] P. P. Matus. The maximum principle and some of its applications. Comput. Meth. Appl. Math., 2(1):50-91, 2002.

[7] P. P. Matus. Monotone schemes of a higher order of accuracy for differential problems with boundary conditions of the second and third kind. Comput. Meth. Appl. Math., 2(4):378-391, 2002.

[8] P. P. Matus. On convergence of difference schemes for ibvp for quasilinear parabolic equation with generalized solutions. Comput. Meth. Appl. Math., 14(3):361-371, 2014.

[9] P. P. Matus, Le Minh Hieu, and L. G. Volkov. Analysis of second order difference schemes on non-uniform grids for quasilinear parabolic equations. Journal of Computational and Applied Mathematics, 2016.

[10] P. P. Matus, V. T. K. Tuyen, and F. J. Gaspar. Monotone difference schemes for linear parabolic equation with mixed boundary conditions. Doklady of the National Academy of Sciences of Belarus., 58(5):18-22, In Russian, 2014.

[11] E. Mitideri and G. Sweers. Weakly coupled elliptic systems and positivity. Mathematische Nachrichten, 173(1):256-286, 1995.

[12] K. M. Morton. Numerical Solution of Convection-Diffusion Problems. London, Chapman \& Hall, 1996.

[13] J.D. Murray. Mathematical Biology II: Spatial Models and Biomedical Applications. Springer Verlag, 2003.

[14] A. Samarskii. The theory of difference schemes. New York, NY: Marcel Dekker, 2001.

[15] A. Samarskii, P. Matus, and P. Vabishchevich. Difference schemes with operator factors. Kluwer Academic Publishers, Boston/Dordrecht/London, 2002.

[16] A. Samarskii, V. Mazhukin, and P. Matus. Difference schemes on non-uniform grids for two-dimensional parabolic equations. Differents. Uravneniya., 34(2):269-280, In Russian, 1998.

[17] A. Samarskii and P. Vabishchevich. Numerical Methods for Solution of Convection-Diffusion Problems. Editorial YRSS, Moskow, 1999 (in Russian). 
[18] A. Samarskii, P. Vabishchevich, and P. Matus. Difference schemes of increased order of accuracy on non-uniform grids. Differents. Uravneniya., 32(2):265-274, In Russian; transl. in Differential Equations, 32 (1996), No. 2, 269-280, 1996.

[19] Boyan Sirakov. Some estimates and maximum principles for weakly coupled systems of elliptic pde. Nonlinear Analysis Theory Methods and Applications, Elsevier, 70(8):3039-3046, 2009. 